\title{
Continental-Scale Evaluation of Remotely Sensed Soil Moisture Products
}

\author{
Wade T. Crow, Member, IEEE, and Xiwu Zhan, Member, IEEE
}

\begin{abstract}
A new data assimilation-based approach for the continental-scale evaluation of remotely sensed surface soil moisture retrievals is applied to four separate soil moisture products over the contiguous U.S. The approach is based on quantifying the ability of a given soil moisture product to correct for known rainfall errors when sequentially assimilated into a simple water balance model. Analysis results provide new insight into the continental-scale performance of surface soil moisture retrieval algorithms based on satellite passive microwave, scatterometer, and thermal remote sensing observations.
\end{abstract}

Index Terms-Microwave radiometry, moisture, rain, remote sensing.

\section{INTRODUCTION}

W ITHIN the past decade, a range of remote sensing techniques have been developed to retrieve surface $(0-2 \mathrm{~cm})$ soil moisture from spaceborne sensors. A variety of sensor types, including geostationary thermal (e.g., [1]), microwave scatterometers (e.g., [2]), and passive microwave radiometers (e.g., [3]), have been utilized in these efforts. However, to date, little is known about either the relative merits of competing approaches or the added benefit for hydrometeorology and hydroclimatology applications of assimilating these observations into a land surface model. Most of this residual uncertainty stems from severe limitations in the availability of ground-based soil moisture observations and the challenge of reliably upscaling point-scale soil moisture measurements to spaceborne footprint scales (typically $>10 \mathrm{~km}$ ) [4].

Recently, Crow [5] introduced an evaluation strategy for remotely sensed soil moisture products that effectively substitutes ground-based rainfall measurements for ground-based soil moisture observations. The approach is based on evaluating the correlation coefficient between antecedent rainfall errors and analysis increments realized during the Kalman filter-based assimilation of remotely sensed soil moisture products into a simple water balance model. Because it does not require ground-based soil moisture observations, the approach enables the spatial expansion of potential soil moisture validation locations from sparse sites containing sufficiently dense groundbased soil moisture measurements to continental-scale regions over which high-quality ground-based rain-gauge information is available [e.g., the entire contiguous U.S. (CONUS) area].

Manuscript received November 28, 2006; revised February 2, 2007. The review of this letter was arranged by Editor E. J. Kim.

W. T. Crow is with the U.S. Department of Agriculture/Agricultural Research Service Hydrology and Remote Sensing Laboratory, Beltsville, 20705 MD USA (e-mail: wade.crow@ars.usda.gov).

$\mathrm{X}$. Zhan is with the National Environmental Satellite, Data, and Information Service, National Oceanic and Atmospheric Administration, Camp Springs, 20746 MD USA.

Digital Object Identifier 10.1109/LGRS.2007.896533
Work in [5] introduced the approach and applied it to four separate passive microwave soil moisture products within eight $1^{\circ}$ latitude/longitude boxes in the southern U.S. This paper expands on these preliminary results by applying the approach to the entire CONUS area $\left(\sim 8001^{\circ}\right.$ boxes) and considering soil moisture products derived from both microwave scatterometer and thermal remote sensing as well as passive microwave radiometry.

\section{DAtA ASSimilation APPROACH}

The approach in [5] is based on using daily satellite-based precipitation estimates $\left(P^{\text {sat }}\right)$ to derive a simple antecedent precipitation index (API) model

$$
\mathrm{API}_{i}=\gamma_{i} \mathrm{API}_{i-1}+P_{i}^{\text {sat }}
$$

where $\gamma$ is the API coefficient, and $i$ is a daily time index. Higher quality daily rainfall accumulations obtained from ground-based gauge networks $\left(P^{\text {gauge }}\right)$ must also be available but are reserved for benchmarking purposes (see Section III). In order to capture evapotranspiration seasonality in a basic way, $\gamma$ in (1) is varied according to day-of-year $(d)$ as

$$
\gamma_{i}=\alpha+\beta \cos \left(2 \pi d_{i} / 365\right) .
$$

The parameters $\alpha$ and $\beta$ are constants set equal to 0.85 and 0.10 (unless otherwise noted).

When available, remotely sensed soil moisture estimates $\theta_{\mathrm{RS}}$ are used to update (1) using a Kalman filter

$$
\mathrm{API}_{i}^{+}=\mathrm{API}_{i}^{-}+K_{i}\left(\theta_{\mathrm{RS}_{i}}-a-b \mathrm{API}_{i}^{-}\right) .
$$

Here, "-" and "+" denote API values before and after Kalman filter updating. Observation operator parameters $a$ and $b$ are derived from least squares regression of API, calculated using $P^{\text {gauge }}$ and no Kalman filter, updating against $\theta_{\mathrm{RS}}$. The Kalman gain $K$ is given by

$$
K_{i}=b T_{i}^{-} /\left(b^{2} T_{i}^{-}+S\right)
$$

where $T$ is the forecast error in API, and $S$ the error in $\theta_{\mathrm{RS}}$ retrievals. At measurement times, $T$ is updated via

$$
T_{i}^{+}=\left(1-b K_{i}\right) T_{i}^{-} .
$$

Between soil moisture measurements and the adjustment of API and $T$ via (3) and (5), the model state API is temporally updated using observed $P^{\text {sat }}$ and (1). In parallel, model forecast error $T$ is updated in time as

$$
T_{i}^{-}=\gamma_{i}^{2} T_{i-1}^{+}+Q
$$


where $Q$ relates to the uncertainty added to an API forecast as it is propagated from time $i-1$ to $i$. Values of $Q$ and $S$ are calibrated through statistical analysis of filter innovations

$$
\nu_{i}=\left(\theta_{\mathrm{RS}_{i}}-a-b \mathrm{API}_{i}^{-}\right) / \sqrt{\left(b^{2} T_{i}^{-}+S\right)} .
$$

Properly constructed linear filters should yield a $\nu$ time series that is serially uncorrelated and has a second moment of one [6]. Here, a simple optimization algorithm is used to iteratively vary $Q$ and $S$ until both constraints are met.

The overall approach goes as follows.

Step 1) Using $P^{\text {gauge }}$ observations and (2), a multiyear integration of (1) is performed without any Kalman filter updating.

Step 2) Based on this integration, estimates of $a$ and $b$ are obtained from least squares regression of results against $\theta_{\mathrm{RS}}$.

Step 3) Using observed $P^{\text {sat }}$ and an assumed value for $Q$, API and $T$ are temporally integrated from initial conditions until the first (next) measurement time using (1) and (6).

Step 4) At measurement times, this forecasted $T$ and an assumed value for $S$ are used to calculate the Kalman gain (4). Which, in turn, is used to update API and $T$ via (3) and (5).

Step 5) Steps 3) and 4) are iteratively repeated in time for the entire length of the $\theta_{\mathrm{RS}}$ time series.

Step 6) The entire Kalman filter loop summarized in Steps 3)-5) is repeated several times until assumed $Q$ and $S$ values are optimized to produce filter innovations $(\nu)$ that are serially uncorrelated and have a second moment of one. The final calibrated iteration of the Kalman filter is used for further analysis.

Of particular emphasis, here, are the updates to API predicted by (3) in the course of assimilating a particular remote sensing product. These updates are commonly referred to as "analysis increments"

$$
\delta_{i}=\mathrm{API}_{i}^{+}-\mathrm{API}_{i}^{-}=K_{i}\left(\theta_{\mathrm{RS}_{i}}-a-b \mathrm{API}_{i}^{-}\right) .
$$

\section{Definition of $R_{\text {value }}$}

Fig. 1 demonstrates how this filtering approach is exploited to evaluate a remotely sensed soil moisture product $\left(\theta_{\mathrm{RS}}\right)$. For a $1^{\circ}$ latitude/longitude box centered at $101.5^{\circ} \mathrm{W}$ and $35.5^{\circ} \mathrm{N}$, Fig. 1(a) plots a daily rainfall time series extracted from both satellite-based $\left(P^{\text {sat }}\right)$ and gauge-based $\left(P^{\text {gauge }}\right)$ rainfall products [derived from the $1^{\circ}$ latitude/longitude daily (1DD) Global Precipitation Climatology Project (GPCP) and Climate Prediction Center (CPC) precipitation datasets, respectively — see Section IV]. Relative to the more accurate $P^{\text {gauge }}$ product, satellite-based $P^{\text {sat }}$ retrievals underestimate rainfall accumulations for a March 19-20, 2003 storm event and overestimate rainfall for an event on March 27-28. As a result of these errors, temporal tendencies in surface soil moisture retrievals obtained from satellite observations are at odds with API predictions derived from $P^{\text {sat }}$. This is observed in Fig. 1(b), where the time series of $\theta_{\mathrm{RS}}$ retrievals (extracted from the AMSRE $_{\mathrm{USDA}}$ product-see Section IV) supports $P^{\text {gauge }}$ (and contradicts $P^{\text {sat }}$ rainfall) in suggesting that the March 19-20, 2003 storm
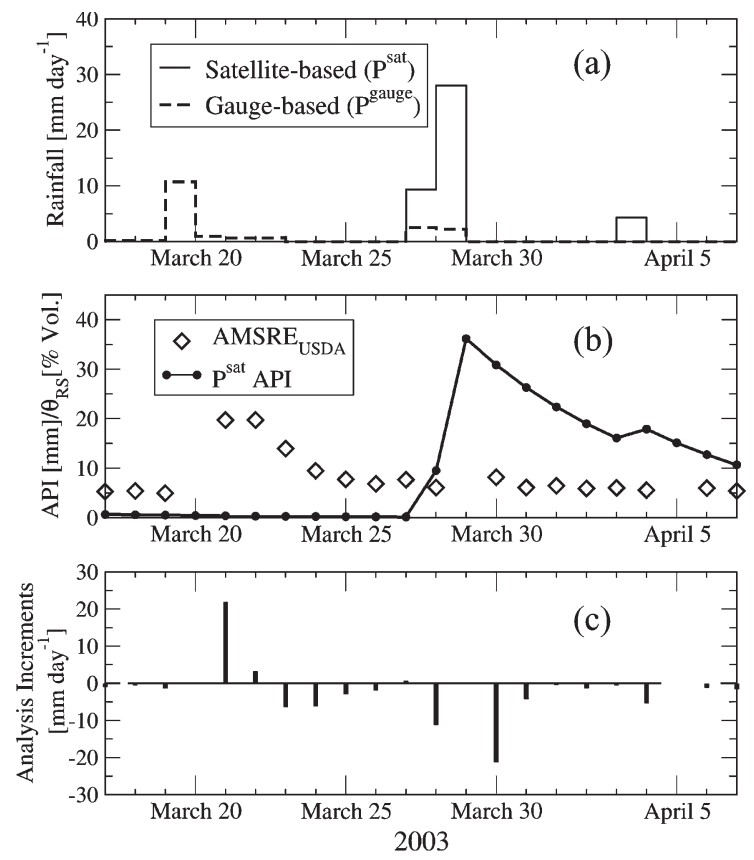

Fig. 1. For a $1^{\circ}$ latitude/longitude box centered at $101.5^{\circ} \mathrm{W}$ and $35.5^{\circ} \mathrm{N}$, time series of (a) daily satellite ( $P^{\text {sat }}$ from GPCP-1DD) and gauge-based ( $P^{\text {gauge }}$ from CPC) rainfall accumulations, $(b) P^{\text {sat }}$-based API (no assimilation) and $\mathrm{AMSRE}_{\mathrm{USDA}}$ soil moisture retrievals, and (c) analysis increments calculated when assimilating AMSRE $_{\mathrm{USDA}}$ into the $P^{\text {sat }}$-based API model.

is the larger of the two events. Consequently, the assimilation of $\theta_{\mathrm{RS}}$ into the $P^{\text {sat }}$-driven API model (using the procedure outlined in Section II) acts to compensate API predictions for the impact of antecedent precipitation errors. Specifically, Kalman filter analysis increments $\delta$ plotted in Fig. 1(c) are inversely correlated with recent rainfall errors plotted in Fig. 1(a).

Fig. 1 implies that, if $\theta_{\mathrm{RS}}$ soil moisture estimates are minimally accurate and the Kalman filter functioning properly, $\delta$ updates should reflect the correction of errors in API predictions resulting from forcing via noisy satellite-based precipitation. In areas of the world where high-quality rainfall observations are available from ground-based gauge networks, such error can be estimated on a daily basis as $\epsilon_{i}^{\text {rain }}=P_{i}^{\text {sat }}-P_{i}^{\text {gauge }}$. To exploit this possibility, Crow [5] suggests temporally aggregating $\delta$ and $\epsilon^{\mathrm{rain}}$ within a series of nonoverlapping windows of length $m$

$$
\begin{gathered}
\overline{\delta_{i}}=\sum_{i+n}^{i+m+n} \delta_{i} \\
\overline{\epsilon_{i}^{\text {rain }}}=\sum_{i}^{i+m} \epsilon_{i}^{\text {rain }}
\end{gathered}
$$

and calculating the standard correlation coefficient $R$ between $\overline{\delta_{i}}$ and $\overline{\epsilon_{i}^{\text {rain }}}$ for a multiyear record of $\theta_{\mathrm{RS}}$. The negative of $R$ is referred to as the $R_{\text {value }}$ coefficient for a particular remotely sensed surface soil moisture product. The lag of $n$ days for $\overline{\delta_{i}}$ in (9) and (10) is introduced to reflect the impact of past rainfall on current soil moisture conditions. Here, values of one and seven day(s) will be used for $n$ and $m$, respectively.

For the period of July 1, 2002-December 31, 2005 and the $1^{\circ}$ box considered in Fig. 1, Fig. 2 plots the (nonoverlapping) seven-day sums of Kalman filter analysis increments $\overline{\delta_{i}}$ calculated when assimilating $\theta_{\mathrm{RS}}$ against seven-day sums of rainfall error $\overline{\epsilon_{i}^{\text {rain }}}$ (lagged in the past by a single day). The $R_{\text {value }}$ 


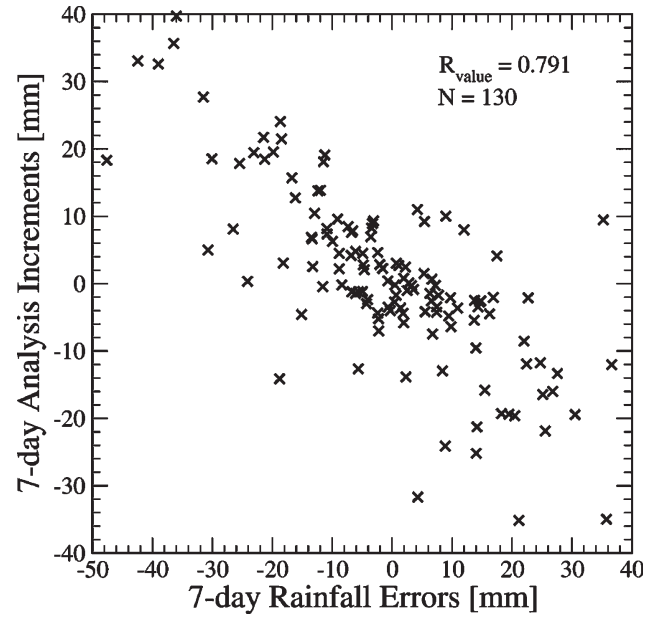

Fig. 2. Scatterplot of seven-day rainfall error total $\left(\overline{\epsilon_{i}^{\text {rain }}}\right)$ versus seven-day sums of Kalman filter analysis increments $\left(\overline{\delta_{i}}\right)$ calculated when assimilating AMSRE $_{\mathrm{USDA}}$ within the $101.5^{\circ} \mathrm{W}$ and $35.5^{\circ} \mathrm{N} 1^{\circ}$ latitude/longitude box between January 1, 2002 and December 31, 2005.

metric is simply the negative of the standard correlation coefficient for this scatterplot. Higher (lower) $R_{\text {value magnitudes }}$ indicate greater (reduced) efficiency in the filtering of past rainfall errors and implies a(n) enhanced (degraded) correlation between $\theta_{\mathrm{RS}}$ and true soil moisture. Consequently, it provides a measure of $\theta_{\mathrm{RS}}$ accuracy obtained without reliance on groundbased soil moisture measurements. In addition, as demonstrated in [5], the same remotely sensed soil moisture product produces progressively higher (lower) $R_{\text {value }}$ magnitudes as the accuracy of $P^{\text {sat }}$ is degraded (improved). This trend reflects the tendency for $\theta_{\mathrm{RS}}$ retrievals of a constant accuracy to become relatively less valuable for land-surface modeling as satellitebased precipitation estimates improve. Consequently, $R_{\text {value }}$ is most accurately interpreted as a proxy of added value, which reflects the ability of $\theta_{\mathrm{RS}}$ retrievals to improve upon a baseline water balance model driven by $P^{\text {sat }}$ observations.

\section{DATA}

As discussed above, the calculation of $R_{\text {value }}$ is based on two separate daily precipitation data sets-a satellite-based global product to force the API model $\left(P^{\mathrm{sat}}\right)$ during the data assimilation analysis and a higher accuracy gauge-based product to function as a benchmark data set in the calculation of precipitation errors $\left(P^{\text {gauge }}\right)$. Here, $P^{\text {sat }}$ is taken from the GPCP-1DD rainfall product based primarily on satellite-based retrievals [7]. $P^{\text {gauge }}$ is obtained from the gauge-based National Center for Environmental Prediction CPC retrospective CONUS rainfall product. Following the approach of the study in [8], CPC data processed as part of the North American Land Data Assimilation System project [9] is aggregated into daily (0 UTZ to $0 \mathrm{UTZ}) 1^{\circ}$ latitude/longitude boxes to match the temporal and spatial attributes of the GPCP-1DD data set. Note that, since the GPCP-1DD rain product is rescaled at coarse time and space scales to match rain-gauge output [7], long-term means of the GPCP-1DD and CPC rainfall datasets (and, therefore, the API predictions derived from them) are approximately equal.

Four separate remotely sensed soil moisture products are considered in the analysis. The first two volumetric soil moisture products are based on Level 2 X-band $(10.6 \mathrm{GHz})$ passive microwave brightness temperature $\left(T_{B}\right)$ observations obtained from the Advanced Microwave Scanning Radiometer (AMSRE) aboard the NASA Aqua satellite. These observations are available with a spatial resolution of about $40^{2} \mathrm{~km}^{2}$ and repeat times of approximately one to two days at CONUS latitudes. The AMSRE ${ }_{\text {NASA }}$ product is the official AMSRE level-three soil moisture product [10] based on the application of the dual-polarization $(\mathrm{H}$ and $\mathrm{V})$ algorithm described in [3] to AMSRE X-band $T_{B}$ observations. In contrast, the AMSRE $_{\text {USDA }}$ product [developed at the U.S. Department of Agriculture (USDA) Hydrology and Remote Sensing Laboratory by T. J. Jackson and X. Zhan] is based on the application of the single-polarization (H only) [11] algorithm to the same AMSRE $T_{B}$ data. The two algorithms differ fundamentally in their approach for estimating vegetation-water-content (VWC) values required for surface soil moisture retrieval from $T_{B}$. The single-polarization AMSRE $\mathrm{USDA}_{\mathrm{S}}$ algorithm uses the Moderate Resolution Imagery Spectrometer 16-day normalized difference vegetation index (NDVI) composite product and the VWC/NDVI regression of [12] to estimate VWC while the AMSRE $_{\text {NASA }}$ approach simultaneously solves for both VWC and surface soil moisture based on the consideration of dualpolarized ( $\mathrm{H}$ and $\mathrm{V}$ ) AMSRE $T_{B}$ observations. All AMSRE results presented here are based on data collected between July 1, 2002 and December 31, 2005.

In addition to soil moisture products derived from the passive microwave AMSRE sensor, surface soil moisture retrievals obtained from European Remote Sensing (ERS)-1 and -2 scatterometer measurements, and the retrieval algorithm as described in [3], are also considered. This product (available online at www.ipf.tuwien.ac.at/radar) has an approximate spatial resolution of $50^{2} \mathrm{~km}^{2}$ and provides a retrieval once every three to four days. The [2] algorithm exploits the multiincidence nature of the ERS scatterometer measurements to correct for vegetation phenology and compares these vegetation corrected values to dry and wet reference backscatter measurements to obtain a relative surface-wetness index. Results are based on ERS-1 and -2 scatterometer measurements obtained between January 1, 1997 and December 31, 2005.

A final measurement strategy for the retrieval of surface soil moisture information is based on the interpretation of variations in thermal-based surface radiometric temperature $\left(T_{R}\right)$ retrievals. The approach in the study of [1] uses Geostationary Environmental Satellite (GOES) observations of $T_{R}$ and the Atmosphere Land Exchange Inverse (ALEXI) model to estimate the ratio between actual and potential soil evaporation. This fraction possesses a close physical relationship with water availability for direct soil evaporation and can be interpreted as a surface soil moisture proxy. ALEXI predicted actual/potential soil-evaporation fractions, subsequently referred to the GOES/ALEXI soil $_{\text {product, are available on a }}$ $10^{2}-\mathrm{km}^{2}$ grid during the 2002-2004 growing seasons (April 1 to October 31). Due to cloud cover, retrieval frequency is limited to once every two or three days.

\section{RESULTS}

The approach outlined in Sections II and III is applied separately for every $1^{\circ}$ latitude/longitude CONUS box and repeated using each of the four remotely sensed soil moisture data sets 

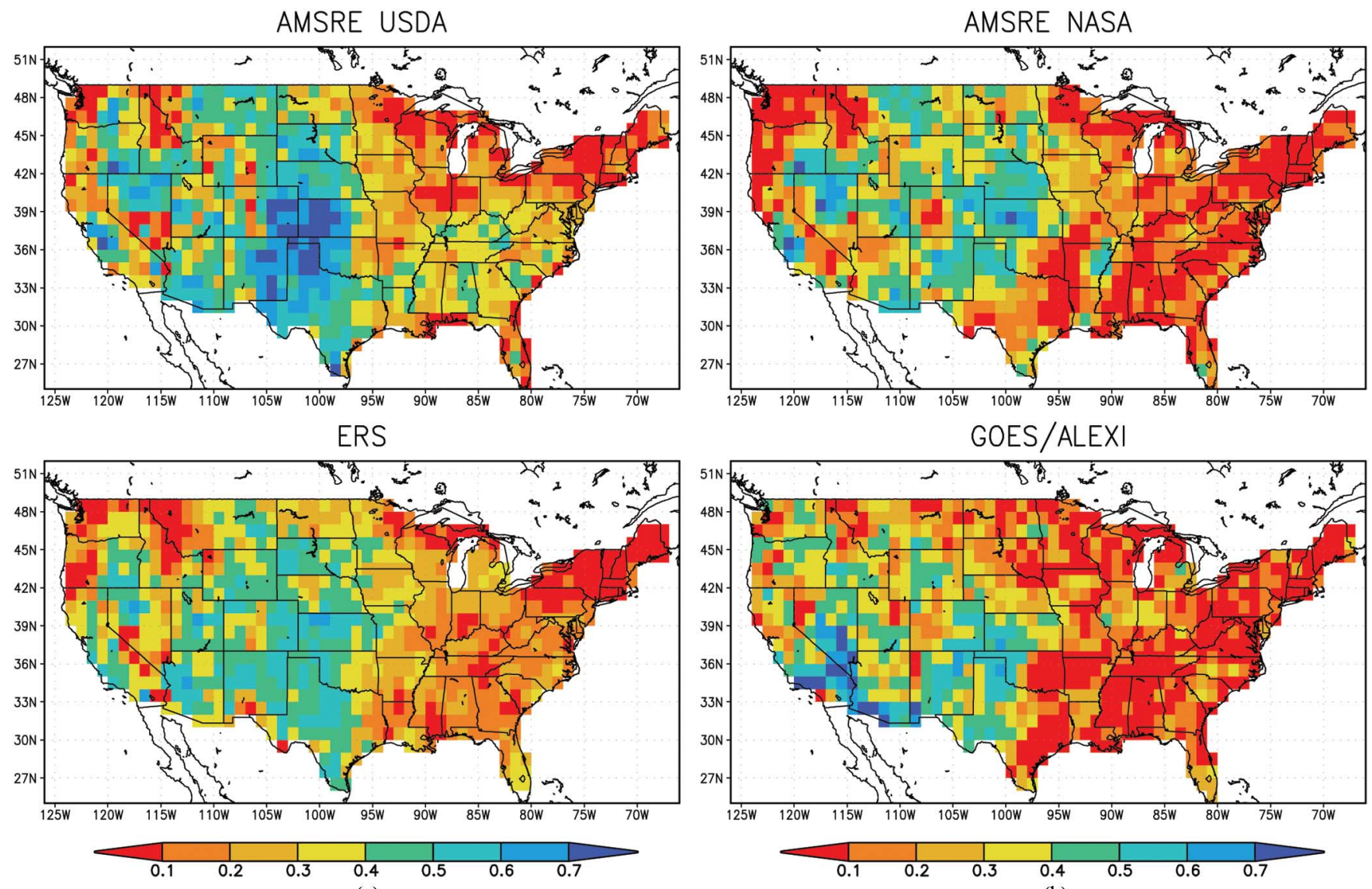

(a)

(b)

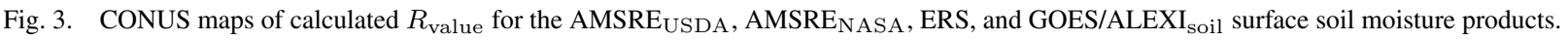

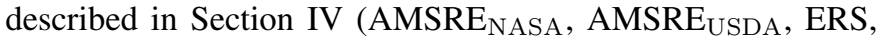

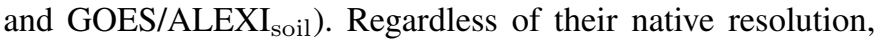
all four products are spatially aggregated to a $1^{\circ}$ resolution to match spatial resolution of the GPCP-1DD $\left(P^{\mathrm{sat}}\right)$ rain product. Observation-operator parameters $a$ and $b$ for each soil moisture product are obtained via a linear least squares line fit separately to the $\theta_{\mathrm{RS}} / \mathrm{API}$ relationship within each $1^{\circ}$ box. Only sevenday periods where at least two remotely sensed soil moisture retrievals are available and at least $2 \mathrm{~mm}$ of total precipitation is reported (in either the $P^{\text {sat }}$ or $P^{\text {gauge }}$ rainfall products) are included in the analysis.

Fig. 3 displays maps of CONUS $R_{\text {value }}$ magnitudes derived in this manner. Spatial patterns of $R_{\text {value }}$ for all products in Fig. 3 mirror basic CONUS vegetation biomes. Reflecting the presence of forest cover, low values are generally noted in the Pacific Northwest $\left(44^{\circ} \mathrm{N}-49^{\circ} \mathrm{N}, 112^{\circ} \mathrm{W}-124^{\circ} \mathrm{W}\right)$, the upper Great Lakes $\left(45^{\circ} \mathrm{N}-49^{\circ} \mathrm{N}, 84^{\circ} \mathrm{W}-95^{\circ} \mathrm{W}\right)$, the Ozark Mountain region $\left(33^{\circ} \mathrm{N}-39^{\circ} \mathrm{N}, 90^{\circ} \mathrm{W}-95^{\circ} \mathrm{W}\right)$, and along a board swath of the eastern U.S. In addition, several differences can be noted between products. For instance, the null hypothesis of nonpositive AMSRE ${ }_{\mathrm{USDA}} R_{\text {value }}$ can be rejected (at $95 \%$ confidence) over $84 \%$ of the CONUS versus $66 \%$ for AMSRE $_{\text {NASA }}$. These percentages represent the fractional CONUS area where soil moisture retrievals are contributing a significant amount of added value to a simple land surface model driven by remotely-sensed precipitation. Most of the fractional difference between the two AMSRE products is attributable to superior
AMSRE $_{\text {USDA }}$ retrievals (i.e., higher $R_{\text {value }}$ ) across the south central and southeastern U.S. (Fig. 3). From an algorithm standpoint, the fundamental difference between the two is the use of ancillary NDVI measurements (and an empirically based regression model) by the single-polarization AMSRE $_{U S D A}$ approach to predict VWC versus the simultaneous retrieval of both soil moisture and VWC based on multipolarization $T_{B}$ by the AMSRE $_{\text {NASA }}$ approach. Since the VWC/NDVI regression model used in the AMSRE USDA $_{\text {algorithm was developed }}$ through field work in rangeland areas of the south central U.S. [12], the superior performance of the AMSRE $\mathrm{USDA}_{\mathrm{USD}}$ product in this region $\left(34^{\circ} \mathrm{N}-40^{\circ} \mathrm{N}, 99^{\circ} \mathrm{W}-105^{\circ} \mathrm{W}\right)$ is not surprising. However, relatively high $R_{\text {value }}$ results for the AMSRE USDA approach within the mixed agricultural/forest areas of the southeastern U.S. suggest that the NDVI/VWC regression model can be successfully applied to other major land-cover types. Rejecting the null hypothesis of zero difference between AMSRE $_{U S D A}$ and AMSRE $_{\mathrm{NASA}}$ at $95 \%$ confidence requires an absolute $R_{\text {value }}$ contrast of approximately 0.2 in Fig. 3 . This threshold is widely met in the south central and southeastern U.S. (Fig. 3). Here, the inclusion of ancillary NDVI measurements for VWC estimates adds detectable value to soil moisture retrievals. In contrast, the marginal impact of NDVI observations is much less along the northern U.S. where $R_{\text {value }}$ differs little between AMSRE USDA $_{\text {and }}$ AMSRE ${ }_{\text {NASA }}$.

Relative to the AMSRE-based products, the ERS scatterometer product demonstrates less spatial variability in response 
to variations in VWC. For instance, note the more gradual decline in $R_{\text {value }}$ for the product when transitioning between lightly vegetated rangeland to forested areas of the southern Great Plains (i.e., moving along a transect at $38^{\circ} \mathrm{N}$ from $\left.105^{\circ} \mathrm{W}-93^{\circ} \mathrm{W}\right)$. This reduction in sensitivity may be due to a slightly lower antenna frequency for ERS observations (6.9 $\mathrm{GHz}$ for ERS versus $10.6 \mathrm{GHz}$ for AMSRE). Overall, the performance of the ERS soil moisture product is roughly on a

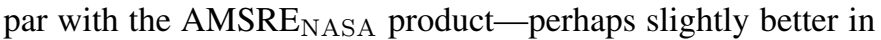
heavily vegetated areas.

The thermal-based GOES/ALEXI soil $_{\text {product demonstrates }}$ the best relative performance in highly water-limited areas of the southwest U.S., where variations in $T_{R}$ are tightly coupled to surface moisture availability $\left(32^{\circ} \mathrm{N}-37^{\circ} \mathrm{N}\right.$, $\left.110^{\circ} \mathrm{W}-120^{\circ} \mathrm{W}\right)$. Despite low levels of VWC, the AMSRE and ERS products do not perform well in this region. Conversely, relative to the microwave-based retrievals, the GOES/ALEXI $_{\text {soil }}$ product performs poorly along the northern edge of the U.S., where evapotranspiration is typically energy limited and surface moisture availability is not strongly coupled with $T_{R}$. In addition to surface soil moisture, thermal-based observations also have potential for inferring deeper root-zone soil moisture information based on the calculation of vegetation fractional evapotranspiration $\left(\mathrm{GOES}_{\mathrm{ALEXI}} \mathrm{veg}\right)$. Under dense

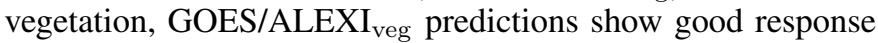
to precipitation on a monthly time scale commensurate with the time scale for root-zone soil water depletion [1]. Unfortunately, $R_{\text {value }}$ reflects only the quick response of shallow $(0-2 \mathrm{~cm})$ soil moisture to daily rainfall and cannot be used to assess retrievals of slower root-zone variations.

Several additional factors can potentially affect the interpretation of Fig. 3. First, the lower observation frequency for ERS and ALEXI soil moisture retrievals (once every two to four days versus every one to two for AMSRE) may reduce $R_{\text {value }}$ magnitudes for ERS soil moisture products even if the intrinsic accuracy of the soil moisture products are equivalent [5]. To control for this effect, $\mathrm{AMSRE}_{\mathrm{USDA}}$ and $\mathrm{AMSRE}_{\mathrm{NASA}} R_{\text {value }}$ results in Fig. 3 were recalculated by randomly subsampling AMSRE-based soil moisture retrievals until their temporal frequency approximated that of the ERS and GOES/ALEXI soil $_{\text {I }}$ products (one measurement every 3.5 days). Such subsampling led to an average relative reduction of $\sim 5 \%$ for AMSRE $\mathrm{ASDA}_{\mathrm{USD}}$ and $\mathrm{AMSRE}_{\mathrm{NASA}} R_{\text {value }}$ magnitudes but did not lead to any qualitative change in results. In addition, sensitivity to $\alpha$ and $\beta$ in (1) was examined by regenerating Fig. 3 for a range of cases based on systematically varying $\alpha$ between 0.80 and 0.95 and $\beta$ between 0.00 and 0.15 . Generally, little sensitivity was found due to either variation. The two noteworthy exceptions to this rule are a tendency for AMSRE $\mathrm{USDA}_{\mathrm{value}} R_{\text {magnitudes in the }}$ southern U.S. to rise as $\beta$ is increased from 0.00 to 0.10 and a trend among all products toward lower $R_{\text {value }}$ magnitudes for $\alpha$ greater than 0.90 .

\section{DISCUSSION}

The data assimilation-based evaluation method of the study in [5] is applied within the CONUS to four separate spaceborne soil moisture products. Results demonstrate the potential for using the approach to evaluate the relative performance of each product within a continental-scale domain. Unlike traditional validation approaches, application of this technique is not limited to extremely sparse space/time periods where sufficiently dense ground-based soil moisture observations are available.

Despite this potential, several points should be considered when interpreting these results. First, $R_{\text {value }}$ magnitudes reflect only the ability of assimilated soil moisture to correct rainfall errors and neglect potential value associated with the correction of additional model shortcomings (e.g., the simple treatment of soil water loss in the API model). In addition, the use of a linear observation operator precludes the representation of potential nonlinearities existing between modeled and retrieved soil moisture [13]. Additional work directed at these limitations may prove useful. Finally, since $R_{\text {value }}$ magnitudes reflect only the ability of a given soil moisture product to correlate with true soil moisture and not their absolute root-mean-square (rms) accuracy, it evaluates products based only on their skill with regards to relative change detection. For land data assimilation applications, such skill is generally regarded as more important than absolute rms retrieval accuracy [14]. However, it should be stressed that this is not uniformly the case for all potential applications.

\section{REFERENCES}

[1] M. C. Anderson, J. M. Norman, J. R. Mecikalski, J. P. Otkin, and W. P. Kustas, "A climatological study of evapotranspiration and moisture stress across the continental U.S. based on thermal remote sensing: II Surface moisture climatology," J. Geophys. Res., 2007, to be published.

[2] W. Wagner, G. Lemoine, and H. Rott, "A method for estimating soil moisture from ERS scatterometer and soil data," Remote Sens. Environ., vol. 70, no. 2, pp. 191-207, Nov. 1999. DOI:10.1016/S00344257(99)00036-X.

[3] E. G. Njoku, T. J. Jackson, V. Lakshmi, T. Chan, and S. V. Nghiem, "Soil moisture retrieval from AMSRE," IEEE Trans. Geosci. Remote Sens., vol. 41, no. 2, pp. 215-229, Feb. 2003.

[4] M. H. Cosh, T. J. Jackson, R. Bindlish, and J. H. Prueger, "Watershed scale temporal persistence of soil moisture and its role in validating satellite estimates," Remote Sens. Environ., vol. 92, no. 4, pp. 427-435, Sep. 2004.

[5] W. T. Crow, "A novel method for quantifying value in spaceborne soil moisture retrievals," J. Hydrometeorol., vol. 8, no. 1, pp. 56-57, Feb. 2007.

[6] A. Gelb, Applied Optimal Estimation. Cambridge, MA: MIT Press, 1974. 374 pp.

[7] G. J. Huffman, R. F. Adler, M. M. Morrissey, D. T. Bolvin, S. Curtis, R. Joyce, B. McGavock, and J. Susskind, "Global precipitation at onedegree daily resolution from multi-satellite observations," J. Hydrometeorol., vol. 2, no. 1, pp. 36-50, Feb. 2001.

[8] J. McPhee and S. Margulis, "Validation and error characterization of GPCP-1DD precipitation product over the contiguous United States," J. Hydrometeorol., vol. 6, no. 4, pp. 441-459, Aug. 2005.

[9] B. A. Cosgrove et al., "Real-time and retrospective forcing in the North American Land Data Assimilation System (NLDAS) project," J. Geophys. Res., vol. 108, no. D22, pp. GCP3.1-GCP3.12, 2003. 8842, DOI:10.1029/2002JD003118.

[10] E. G. Njoku, AMSR-E/Aqua Daily L3 Surface Soil Moisture, Interpretive Parameters, and QC EASE-Grids, July 2002 to December 2005. Boulder, CO: Nat. Snow and Ice Data Center, 2006. Daily updated digital media.

[11] T. J. Jackson, "Measuring surface soil moisture using passive microwave remote sensing," Hydrol. Process., vol. 7, no. 2, pp. 139-152, 1993.

[12] T. J. Jackson, D. M. Le Vine, A. Y. Hsu, A. Oldak, P. J. Starks, C. T. Swift, J. Isham, and M. Haken, "Soil moisture mapping at regional scales using microwave radiometry: The Southern Great Plains Hydrology Experiment," IEEE Trans. Geosci. Remote Sens., vol. 37, no. 5, pp. 21362151, Sep. 1999.

[13] M. Drusch, E. F. Wood, and H. Gao, "Observation operators for the direct assimilation of TRMM microwave imager retrieved soil moisture," Geophys. Res. Lett., vol. 32, no. 15, pp. L15403.1-L15403.4, 2005. DOI:10.1029/2005GL023623.

[14] W. T. Crow, R. D. Koster, R. H. Reichle, and H. O. Sharif, "Relevance of time-varying and time-invariant retrieval error sources on the utility of spaceborne soil moisture products," Geophys. Res. Lett., vol. 32, no. 24, pp. L24405.1-L24405.5, 2005. DOI:10.1029/2005GL024889. 